\title{
Causality of black holes in 4-dimensional Einstein-Gauss-Bonnet-Maxwell theory
}

\author{
Xian-Hui Ge ${ }^{1,3, a}$, Sang-Jin Sin ${ }^{2, b}$ \\ ${ }^{1}$ Department of Physics, Shanghai University, Shanghai 200444, China \\ ${ }^{2}$ Department of Physics, Hanyang University, Seoul 133-791, South Korea \\ ${ }^{3}$ Center for Gravitation and Cosmology, College of Physical Science and Technology, Yangzhou University, Yangzhou 225009, China
}

Received: 20 May 2020 / Accepted: 26 July 2020 / Published online: 3 August 2020

(C) The Author(s) 2020

\begin{abstract}
We study charged black hole solutions in 4dimensional (4D) Einstein-Gauss-Bonnet-Maxwell theory to the linearized perturbation level. We first compute the shear viscosity to entropy density ratio. We then demonstrate how bulk causal structure analysis imposes an upper bound on the Gauss-Bonnet coupling constant in the AdS space. Causality constrains the value of Gauss-Bonnet coupling constant $\alpha_{G B}$ to be bounded by $\alpha_{G B} \leq 0$ as $D \rightarrow 4$.
\end{abstract}

\section{Introduction}

The AdS/CFT correspondence [1-3] provides a powerful tool for studying the physics of strongly coupled gauge theories and can also be used to examining alternative theories to the general relativity. Alternative theories to Einstein's General Relativity paradigm can be scrutinized by diverse approaches. The higher derivative gravity with $\alpha^{\prime}$ corrections was also studied widely within the framework of the AdS/CFT correspondence (see [4-12] for an incomplete list). For Einstein-Gauss-Bonnet (EGB) theory, strong constraints can be imposed on the Gauss-Bonnet coupling constant from the analysis of the bulk causal structure. For 5-dimensional (5D) EGB theory, causality demands $\alpha_{G B} \leq$ 0.09 to avoid superluminal propagation of signals in the dual boundary field theory [5-7, 12]. Recently, revived interests on EGB gravity in 4-dimensional spacetime have been first concerned in [13].

The 4-dimensional EGB gravity is realized by first rescaling the coupling constant $\alpha^{\prime} \rightarrow \frac{\alpha^{\prime}}{D-4}$ of the Gauss-Bonnet term and then take the limit $D \rightarrow 4$ [13]. In this way, the Lovelock's theorem [14-16] can be bypassed and spheri-

\footnotetext{
a e-mail: gexh@shu.edu.cn (corresponding author)

b e-mail: sjsin@hanyang.ac.kr
}

cally symmetric 4D black hole solutions can be obtained in the presence of the Gauss-Bonnet term.

In this paper, we are going to investigate whether causality violation happens in the $4 D$ EGB gravity and check the upper bound of the Gauss-Bonnet coupling constant. As shown in $[5-7,9]$, higher derivative terms in the gravity action can result in superluminal propagation of gravitons outside the light cone of a given background geometry. The graviton cone in such case does not coincide with the standard null cone or light cone defined by the background metric. Utilizing the tool provided by AdS/CFT correspondence, firstly we will study the linearized perturbation of the black holes in 4D Einstein-Gauss-Bonnet-Maxwell (EGBM) gravity. Then we calculate the shear viscosity to entropy density ratio in this context. We then examine the causality constraint on the Gauss-Bonnet coupling constant. We are going to show that for 4D black holes in EGBM theory, if the limit $D \rightarrow 4$ is taken after the series expansion of the local speed of the transverse graviton near the boundary, no causality violation requires $\alpha_{G B} \leq 0$. This somehow agrees with the results obtained in [17], positive Gauss-Bonnet coupling from the EGB theory compactified to $4 \mathrm{D}$ on a circle rendering the merger of two black holes violating the second law of thermodynamics.

We will show that the bulk graviton propagating faster than the local speed of light could lead to signals in the boundary theory propagating outside the light cone. According to the AdS/CFT correspondence, the boundary theory is nongravitational. In a boosted frame, perturbations will propagate backward in time. Hence, these could lead to unambiguous signals of causality violation.

The structure of this paper is organized as follows. In Sect. 2, we study the charged black hole solutions in 4D Einstein-Gauss-Bonnet-Maxwell theory. Then, in Sect. 3, we compute the shear viscosity to entropy density ratio. In Sect. 4, we discuss the bulk causal structure and its boundary 
consequences. We also compare our results with causality constraints on higher derivative gravity corrections to the graviton three-point coupling. The conclusion and discussions are provided in the last section.

\section{Charged black hole solutions in Einstein-Gauss-Bonnet-Maxwell theory}

We now consider the Einstein-Maxwell Gauss-Bonnet in $\mathrm{D}$ dimensions with a negative cosmological constant $\Lambda_{0}=$ $-\frac{(D-1)(D-2)}{l^{2}}$ given by the action

$I=\frac{1}{16 \pi} \int \mathrm{d}^{D} x \sqrt{-g}\left(R-2 \Lambda_{0}+\frac{\alpha^{\prime}}{D-4} \mathcal{G}-F_{\mu \nu} F^{\mu \nu}\right)$,

where $\alpha^{\prime}$ is a (positive) Gauss-Bonnet coupling constant with dimension (length) ${ }^{2}$, the field strength is defined as $F_{\mu \nu}(x)=$ $\partial_{\mu} A_{\nu}(x)-\partial_{\nu} A_{\mu}(x)$ and $\mathcal{G}=\left(R_{\mu \nu \rho \sigma} R^{\mu \nu \rho \sigma}-4 R_{\mu \nu} R^{\mu \nu}+\right.$ $\left.R^{2}\right)$. The general D-dimensional static and maximally symmetric black hole can be described as

$d s^{2}=-f(r) d t^{2}+\frac{d r^{2}}{f(r)}+r^{2} d \Omega_{2, \kappa}^{2}, \quad d \Omega_{2, \kappa}^{2}=\frac{d x^{2}}{1-\kappa x^{2}}+x^{2} d \varphi^{2}$,

and an electrostatic vector potential

$A_{t}=V(r) d t$

where $\kappa=-1,0,1$. Since all the functions are radially dependent only, by substituting (2.2) into the action, we obtain

$V^{\prime}(r)=-\frac{Q}{r^{D-2}}$,

with the integral constant $Q$ as the electric charge. The metric function $f(r)$ can be obtained by defining a new variable $\psi(r)$

$f(r) \equiv \kappa-r^{2} \psi(r)$.

In this form, the action reduces to

$$
\begin{aligned}
I= & \frac{\Omega_{d-2}}{16 \pi} \int d t d r(D-2)\left[r^{D-1} \psi\left(1+\alpha^{\prime}(D-3) \psi\right)\right. \\
& \left.+\frac{r^{D-1}}{l^{2}}+\frac{2 Q^{2} r^{3-D}}{(D-3)(D-2)}\right]^{\prime}
\end{aligned}
$$

with $\Omega_{d-2}=\frac{2 \pi \frac{D-2}{2}}{\Gamma\left(\frac{D-1}{2}\right)}$. Notice that $\psi(r)$ satisfies the relation [18-20]

$$
\begin{aligned}
\psi & +\alpha^{\prime}(D-3) \psi^{2} \\
& =\frac{16 \pi M}{(D-2) r^{D-1} \Omega_{d-2}}-\frac{1}{l^{2}}-\frac{2 Q^{2} r^{4-2 D}}{(D-3)(D-2)},
\end{aligned}
$$

where $M$ is the ADM mass. We then obtain the metric function and the scalar potential as follows

$$
\begin{aligned}
& f(r)=\kappa-\frac{r^{2}}{2 \alpha^{\prime}(D-3)} \\
& {\left[-1 \pm \sqrt{1-4(D-3) \alpha^{\prime}\left(\frac{1}{l^{2}}+\frac{2 Q^{2} r^{4-2 D}}{(D-3)(D-2)}-\frac{16 \pi M r^{1-D}}{(D-2) \Omega_{D-2}}\right)}\right],} \\
& A_{t}=-\frac{Q}{(D-3) r^{D-3}} d t .
\end{aligned}
$$

The sign " + " denotes the perturbative branch in $\alpha$, while the "- -" sign corresponds to the branch that the metric function $f(r)$ goes to infinity as $\alpha^{\prime} \rightarrow 0$. We choose the " + " sign hereafter. A rigorous method of compactifying EGB gravity on a $(D-4)$-dimensional maximally symmetric space was introduced in [21]. The thermodynamics and geometric properties of the 4D Einstein-Gauss-Bonnet black holes have been studied in several papers [22-38]. In what follows, we focus on the black hole solution with the planar horizon by taking $\kappa=0$.

For planar black branes in AdS space, the line elements can be written as

$$
\begin{aligned}
d s^{2}= & -H(r) N^{2} d t^{2}+H^{-1}(r) d r^{2} \\
& +\frac{r^{2}}{l^{2}} d x^{i} d x^{i}, \text { with } i=1, \ldots, D-2,
\end{aligned}
$$

where

$$
\begin{aligned}
H(r) & =\frac{r^{2}}{2 \alpha_{G B} l^{2}}\left[1-\sqrt{1-4 \alpha_{G B}\left(1-\frac{m l^{2}}{r^{D-1}}+\frac{q^{2} l^{2}}{r^{2 D-4}}\right)}\right] \\
& =\frac{r^{2}}{2 \alpha_{G B} l^{2}}\left[1-\sqrt{1-4 \alpha_{G B}\left(1-\frac{r_{+}^{D-1}}{r^{D-1}}-a \frac{r_{+}^{D-1}}{r^{D-1}}+a \frac{r_{+}^{2 D-4}}{r^{2 D-4}}\right)}\right] .
\end{aligned}
$$

Note that $\alpha_{G B}$ and $\alpha^{\prime}$ are connected by a relation $\alpha_{G B}=(D-$ 3) $\alpha^{\prime} / l^{2}, a=\frac{q^{2} l^{2}}{r_{+}^{2 D-4}}$ denotes dimensionless charge parameter and the parameter $l$ corresponds to AdS radius. The horizon is located at $r=r_{+}$. The gravitational mass $M$ and the charge $Q$ are expressed as

$$
\begin{aligned}
M & =\frac{(D-2) \Omega_{D-2}}{16 \pi} m, \\
Q^{2} & =\frac{(D-2)(D-3)}{2} q^{2} .
\end{aligned}
$$

Taken the limit $\alpha^{\prime} \rightarrow 0$, the solution recovers the metric of Reissner-Nordström-AdS black branes.

The constant $N^{2}$ in the metric (2.9) can be determined at the boundary whose geometry would reduce to the flat 
Minkowski metric conformaly, i.e. $\mathrm{d} s^{2} \propto-c^{2} \mathrm{~d} t^{2}+\mathrm{d} \mathbf{x}^{2}$. On the boundary with $r \rightarrow \infty$, we have

$H(r) N^{2} \rightarrow \frac{r^{2}}{l^{2}}$,

so that $N^{2}$ is fixed as

$N^{2}=\frac{1}{2}\left(1+\sqrt{1-4 \alpha_{G B}}\right)$.

Note that the boundary speed of light is specified to be unity $c=1$.

The Hawking temperature at the event horizon is given by

$T=\frac{1}{2 \pi \sqrt{g_{r r}}} \frac{\mathrm{d} \sqrt{g_{t t}}}{\mathrm{~d} r}=\frac{N r_{+}}{4 \pi l^{2}}[(D-1)-(D-3) a]$.

The black brane approaches extremal as $a \rightarrow \frac{D-1}{D-3}$ (i.e. $T \rightarrow$ $0)$. The entropy density is given by [18-20]

$s=\frac{1}{4} \frac{r_{+}^{D-2}}{l^{D-2}}$.

In order to investigate the causality structure and the upper bound of the Gauss-Bonnet coupling constant in fourdimensional spacetime, we will take the $D \rightarrow 4$ limit and analysis the shear viscosity to entropy density ratio first.

\section{Shear viscosity}

In this section, we are going to study the shear viscosity in the 4D Einstein-Maxwell Gauss-Bonnet gravity theory and examine the shear viscosity bound. Since we already took $\alpha^{\prime} \rightarrow \frac{\alpha^{\prime}}{D-4}$ in Eq. (2.1), we will compute the shear viscosity in general $D$ dimensions and then take the limit $D \rightarrow 4$ so as to circumvent the Lovelock theorem. It is convenient to introduce new coordinates in the following computation

$$
\begin{aligned}
& z=\frac{r}{r_{+}}, \quad \omega=\frac{l^{2}}{r_{+}} \bar{\omega}, \quad k_{3}=\frac{l^{2}}{r_{+}^{2}} \bar{k}_{3}, \quad f(z)=\frac{l^{2}}{r_{+}^{2}} H(r), \\
& f(z)=\frac{z^{2}}{2 \alpha_{G B}}\left[1-\sqrt{1-4 \alpha_{G B}\left(1-\frac{a+1}{z^{D-1}}+\frac{a}{z^{2 D-4}}\right)}\right] .
\end{aligned}
$$

We now study the tensor type perturbation $h_{x_{j}}^{x_{i}}\left(t, x_{i}, z\right)=$ $\phi\left(t, x_{i}, z\right)$ with $i \neq j$ on the black brane background of the form

$$
\begin{aligned}
d s^{2}=- & f(z) N^{2} \mathrm{~d} t^{2}+\frac{\mathrm{d} z^{2}}{b^{2} f(z)}+\frac{z^{2}}{b^{2} l^{2}}\left(2 \phi\left(t, x_{i}, z\right) \mathrm{d} x_{i} \mathrm{~d} x_{j}\right. \\
& \left.+\sum_{i=1}^{D-2} \mathrm{~d} x_{i}^{2}\right),
\end{aligned}
$$

where $b=\frac{1}{r_{+}^{2}}$. Using Fourier decomposition

$\phi\left(t, x_{i}, z\right)=\int \frac{\mathrm{d}^{D-1} k}{(2 \pi)^{D-1}} \mathrm{e}^{-i \bar{\omega} t+i \bar{k}_{i} x_{i}} \phi(k, z)$,

we can obtain the equation of motion for $\phi(z)$ from the Einstein-Gauss-Bonnet-Maxwell field equation

$\partial_{z}\left(\mathcal{N}^{z x_{i}} \partial_{z} \phi\right)+\omega^{2} \mathcal{N}^{t x_{i}} \phi-k_{i}^{2} \mathcal{N}^{x_{i} x_{j}} \phi=0$,

where

$$
\begin{aligned}
& \mathcal{N}^{z x_{i}}=\frac{1}{16 \pi} \sqrt{-g} g^{x_{i} x_{i}} g(z), \\
& \mathcal{N}^{t x_{i}}=-\frac{1}{16 \pi} \sqrt{-g} g^{t t} g(z), \\
& \mathcal{N}^{x_{i} x_{j}}=\frac{1}{16 \pi} \sqrt{-g} g^{x_{i} x_{i}} g_{2}(z), \\
& g(z)=1-\frac{2 \alpha_{G B}}{D-3}\left[z^{-1} f^{\prime}+z^{-2}(D-5) f\right], \\
& g_{2}(z)=1-\frac{2 \alpha_{G B}}{(D-3)(D-4)} \\
& \left(f^{\prime \prime}+(D-5)(D-6) z^{-2} f+2(D-5) z^{-1} f^{\prime}\right),
\end{aligned}
$$

and the prime denotes the derivative with respect to $z$. Note that the factors $(D-5)$ and $(D-6)$ in the expression of $g_{2}(z)$ comes from higher than 5-dimensional contribution of the Gauss-Bonnet theory.

The Green function related to the shear viscosity takes the form

$G_{x_{i} x_{j}, x_{i} x_{j}}=\frac{\mathcal{N}^{z x_{i}} \partial_{z} \phi}{\phi}$.

The shear viscosity can be defined as

$\eta_{x_{i} x_{j}, x_{i} x_{j}}=\frac{-G_{x_{i} x_{j}, x_{i} x_{j}}}{i \omega}$.

We can then recast Eq. (3.15) as a flow equation

$\partial_{z} \eta_{x_{i} x_{j}, x_{i} x_{j}}=\left(\frac{\eta_{x_{i} x_{j}, x_{i} x_{j}}^{2}}{\mathcal{N}^{z x_{i}}}-\mathcal{N}^{t x_{i}}\right)+\frac{i}{\omega} \mathcal{N}^{x_{i} x_{j}} k_{i}^{2}$.

The shear viscosity can be computed by requiring horizon regularity

$$
\begin{aligned}
& \eta_{x_{i} x_{j}, x_{i} x_{j}}=\left.\left(\mathcal{N}^{z x_{i}} \mathcal{N}^{t x_{i}}\right)\right|_{z=1} \\
& \quad=\frac{1}{16 \pi}\left(\frac{r_{+}^{D-2}}{l^{D-2}}\right)\left(1-\frac{2 \alpha_{G B}}{(D-3)}[(D-1)-(D-3) a]\right) .
\end{aligned}
$$

The ratio of the shear viscosity to the entropy density for 4D charged black hole solutions in Gauss-Bonnet gravity is then 
$\frac{\eta_{x_{i} x_{j}, x_{i} x_{j}}}{s}=\frac{1}{4 \pi}\left(1-\frac{2 \alpha_{G B}}{(D-3)}[(D-1)-(D-3) a]\right)$.

In the limit $D \rightarrow 4$, we obtain

$\frac{\eta_{x_{i} x_{j}, x_{i} x_{j}}}{s}=\frac{1}{4 \pi}\left[1-2 \alpha_{G B}(3-a)\right]$.

We can see that for 4D Einstein-Gauss-Bonnet theory, the shear viscosity bound can still be violated. But as the black hole temperature approaches zero $a \rightarrow 3$, one can recover the well-known result $\eta / s \sim 1 / 4 \pi[9,39-46]$.

\section{Bulk causal structure}

According to the AdS/CFT correspondence, the physics in bulk 4D AdS gravity is dual to boundary 3D quantum field theory on its boundary. In this section, we study the bulk causal structure and show how a high-momentum metastable state in the bulk graviton wave equation that may have a consequence for boundary causality.

Because of higher derivative terms in the gravity action, Eq. (3.15) for the propagation of a transverse graviton differs from the standard Klein-Gordon equation of a minimally coupled massless scalar field propagating in the same background geometry. Writing the wave function of the transverse graviton as

$\phi\left(x_{i}, z\right)=\mathrm{e}^{-i \omega t+i k z+i k_{i} x_{i}}$

and taking large momenta limit $k^{\mu} \rightarrow \infty$, one can find that the equation of motion (3.15) reduces to

$k^{\mu} k^{\nu} g_{\mu \nu}^{\text {eff }} \simeq 0$

where the effective metric is

$\mathrm{d} s_{\text {eff }}^{2}=g_{\mu \nu}^{\text {eff }} \mathrm{d} x^{\mu} \mathrm{d} x^{\nu}=N^{2} f(r)\left(-\mathrm{d} t^{2}+\frac{1}{c_{g}^{2}} \mathrm{~d} x_{i}^{2}\right)+\frac{1}{f(r)} \mathrm{d} r^{2}$.

Note that the function $c_{g}$ can be interpreted as the local speed of graviton on a constant $r$-hypersurface $[7,47]$ :

$$
\begin{aligned}
& c_{g}^{2}(z) \\
& \quad=\frac{N^{2} f}{z^{2}} \frac{1-\frac{2 \alpha_{G B}}{(D-3)(D-4)}\left(f^{\prime \prime}+(D-5)(D-6) z^{-2} f+2(D-5) z^{-1} f^{\prime}\right)}{1-\frac{2 \alpha_{G B}}{(D-3)}\left[z^{-1} f^{\prime}+z^{-2}(D-5) f\right]},
\end{aligned}
$$

where the prime denotes derivative with respect to $r$ and $z=r / r_{+}$. The local speed of light defined by the background metric $c_{b}^{2}=\frac{N^{2} f(z)}{z^{2}}$, which is 1 at the boundary $z \rightarrow \infty$. In the bulk, the background local speed of light $c_{b}$ is smaller than 1 because of the redshift of the black hole geometry.

The causality problem arises because a graviton wave packet moving at speed $c_{g}$ in the bulk corresponds to perturbations of the stress tensor propagating with the same velocity in the boundary theory. One approach to the limit $D \rightarrow 4$ is to expand $c_{g}^{2}$ near the boundary $z \rightarrow \infty$ for general $D$ [7]

$$
\begin{aligned}
c_{g}^{2}-1= & -\frac{\left(D^{2}-5 D+10\right)(1+a)}{2(D-3)(D-4)} \\
& +\frac{(D-1)(1+a)}{(D-3)(D-4)\left(1-4 \alpha_{G B}\right)} \\
& \left.-\frac{1+a}{2 \sqrt{1-4 \alpha_{G B}}}\right) \frac{1}{z^{D-1}}+\mathcal{O}\left(z^{-D}\right) .
\end{aligned}
$$

The condition that the local speed of graviton should be smaller than 1 requires

$$
\begin{aligned}
& -\frac{\left(D^{2}-5 D+10\right)}{2(D-3)(D-4)}+\frac{(D-1)}{(D-3)(D-4)\left(1-4 \alpha_{G B}\right)} \\
& -\frac{1}{2 \sqrt{1-4 \alpha_{G B}}} \leq 0 .
\end{aligned}
$$

Note that this is significantly different from the result obtained in [7]

$\alpha_{G B} \leq \frac{D^{4}-10 D^{3}+41 D^{2}-92 D+96}{4\left(D^{2}-5 D+10\right)^{2}}$,

which in the $D \rightarrow 4$ limit leads to $\alpha_{G B} \leq 0$. But as $D=5$, one can recover the well-known result $\alpha_{G B} \leq 0.09$ [6].

The geodesic equation of motion $g_{\mu \nu} \frac{\text { eff } \frac{d}{d} d s}{d s} \frac{d x^{\nu}}{d s}=0$ describes the propagation of graviton in the bulk. In order to compute the Shapiro time delay of the graviton, one can define energy $\omega$ and momentum $k$ as conserved integrals of motion along the geodesic

$\omega=N^{2} f(r) \frac{d t}{d s}, \quad k=\frac{N^{2} f(r)}{c_{g}^{2}} \frac{d x_{i}}{d s}$.

The geodesic equation can then be recast as

$\frac{d r}{d \bar{s}}=\alpha^{2}-c_{g}^{2}, \quad \alpha^{2}=\omega^{2} / k^{2}$,

where $\bar{s}=k s / N$. This equation describes the radial motion of a test particle with energy $\alpha^{2}$ in an effective potential $c_{g}^{2}$. The Shapiro time delay can be evaluated as

$\Delta t=2 \int_{r_{0}}^{\infty} \frac{d t}{d \bar{s}} \frac{d \bar{s}}{d r} d r=\frac{2}{N} \int_{r_{0}}^{\infty} d r \frac{\alpha}{f \sqrt{\alpha^{2}-c_{g}^{2}}}$.

To calculate the local speed of the graviton, we need to compute the boundary displacement of a graviton after it bounces back from the bulk 
$\Delta x_{i}=2 \int_{r_{0}}^{\infty} \frac{d x_{i}}{d \bar{s}} \frac{d \bar{s}}{d r} d r=\frac{2}{N} \int_{r_{0}}^{\infty} d r \frac{c_{g}^{2}}{f \sqrt{\alpha^{2}-c_{g}^{2}}}$.

If $c_{g}>1$, the geodesic line which starts from the spatial boundary can bounce back to the boundary. The turning point locates at $\alpha^{2}=c_{g}^{2}$. The microcausality in the boundary CFT can be violated when a bouncing graviton geodesic satisfies $\frac{\Delta x_{i}}{\Delta t}>1$. The Shapiro time delay of an energetic graviton through the bulk can also be calculated in the shock wave background [48]. The causality from the graviton three-point vertex implies a constraint on the conformal anomaly coefficients $\left|\frac{a-c}{c}\right| \lesssim \frac{1}{\Delta_{\text {gap }}^{2}}[48]$, where $\alpha_{G B} \propto \frac{a-c}{c}$ and $\Delta_{\text {gap }}$ the dimension of the lightest higher spin single trace operator. ${ }^{1}$

The bulk causal structure and its relation with the boundary theory can be discussed as follows. In the boundary theory, the local operators create bulk disturbances at infinity that propagate along the graviton geodesics deep inside the bulk. The equation of motion for $\phi$ in (3.15) can be interpreted as an equation describing metastable quasiparticles of the boundary field theory. Now, we recast the equation of motion of the wave function (3.15) in a Schrödinger form,

$$
-\frac{d^{2} \psi}{d r_{*}^{2}}+V\left(z\left(r_{*}\right)\right) \psi=\omega^{2} \psi, \quad \frac{d r_{*}}{d z}=\frac{1}{N f(z)},
$$

where $\psi\left(z\left(r_{*}\right)\right)$ and the potential are defined by

$$
\begin{aligned}
\psi= & K(z) \phi, \quad K(z) \equiv \sqrt{\frac{g(z)}{z^{D-2} f(z)},} \\
V= & k^{2} c_{g}^{2}+V_{1}(z), \\
V_{1}(z) \equiv & N^{2}\left[\left(f(z) \frac{\partial \ln K(z)}{\partial z}\right)^{2}\right. \\
& \left.+f(z) \frac{\partial}{\partial z}\left(f(z) \frac{\partial \ln K(z)}{\partial z}\right)\right] .
\end{aligned}
$$

Geodesics starting from the boundary can bounce back to the boundary. It has been proven that the quasiparticles can travel faster than the speed of light and violate causality [7]. From the geodesic equation of motion

$g_{\mu \nu}^{\text {eff }} \frac{\mathrm{d} x^{\mu}}{\mathrm{d} s} \frac{\mathrm{d} x^{\nu}}{\mathrm{d} s}=0$

and the Bohr-Sommerfield quantization condition

$\int \mathrm{d} r_{*} \sqrt{\omega^{2}-k^{2} c_{g}^{2}}=\left(n-\frac{1}{4}\right) \pi$,

one can find that the group velocity of the test particle along the geodesic line is given by [6]

$v_{g}=\frac{\mathrm{d} \omega}{\mathrm{d} k} \rightarrow c_{g}$

\footnotetext{
${ }^{1}$ It was demonstrated that in flat space the positivity of the time delay implies that the purely Gauss-Bonnet theory is acausal [48]. Also for the Gauss-Bonnet theory compactified to four dimensions on a circle, positive $\alpha_{G B}$ infers that a merger of two black holes can violate the second law while negative $\alpha_{G B}$ can make black hole entropy negative [17].
}

The Eq. (4.29) implies that causality imposes the condition $\alpha_{G B} \leq 0$ to avoid propagation of signals faster than the speed of light. A natural question is whether $\alpha_{G B}$ can be negative from the holographic point of view. For black holes in $5 \mathrm{D}$ EGB gravity, $\alpha_{G B}$ has a lower bound $\alpha_{G B} \geq-7 / 36$ from the analysis of sound mode perturbations [12]. We leave the study on sound mode perturbations in 4D EGB gravity theory to future studies.

Another interesting question deserves study is the contribution of the 4D Gauss-Bonnet term to three point functions of gravitons [48]. The weakly coupled gravity theory in the tree-level approximation can reduce to the Einstein gravity theory at long distances. However, there are possible higher derivative corrections in the intermediate regimes that at low enough but still weakly coupled. Among those corrections, the graviton-three point coupling is the simplest possible correction. The universality of the three-graviton coupling is also a fundamental property of quantum gravity. The causality problem is closely related to the on-shell three-point functions of the weakly coupled gravity for the reason that the three-point functions determine the time delay. It was shown in [48] that in a theory with up to spin 2 particles the causality problem can only be solved when $\alpha_{4}=0$, where $\alpha_{4}$ is the coupling of the perturbations of the general form of the second order of the gravity: $\frac{\alpha_{4}}{2}\left[\nabla_{(\mu} \nabla_{\nu)} R^{\alpha \beta \rho \sigma}\right] \delta R_{\alpha \beta \rho \sigma}$ ( see also $[49,50]$ for related discussions).

\section{Conclusions and discussions}

In summary, we studied the linearized metric perturbation of black holes in 4D Einstein-Gauss-Bonnet-Maxwell theory within the framework of the AdS/CFT correspondence. The charged black hole solutions were obtained for general $D$ dimensions. We then study the shear viscosity to entropy density ratio by considering the planar black brane solution. The ratio $\eta_{x_{i} x_{j}, x_{i} x_{j}} / s=\frac{1}{4 \pi}\left[1-2 \alpha_{G B}(3-a)\right]$ turns out to be different from the Kovtun-Son-Starinets bound $\eta / s=1 / 4 \pi$ if $\alpha_{G B}$ is non-vanishing. We then investigated the bulk causal structure of the 4D charged black holes. In order to guarantee no violation of causality in the boundary field, the Gauss-Bonnet coupling $\alpha_{G B}$ should be in the range $\alpha_{G B} \leq 0$. Note that these results were obtained by following the procedure proposed in [13]: First define the Gauss-Bonnet coupling $\alpha^{\prime} \rightarrow \frac{\alpha^{\prime}}{D-4}$ and then take $D \rightarrow 4$ limit. The results obtained here is comparable and consistent with the causality constraint on corrections to the graviton three-point coupling obtained in [48-50]. The contribution of this modified EGB term to the three-point function of gravitons deserves further studies. However, we would like to carry out the corresponding discussions in which a mathematically more rigorous definition for the $D \rightarrow 4$ limit of EGB gravity is well established. 
If one adds a linear axion field into the action (2.1) and breaks the translational symmetry, then the bulk causal structure could be drastically changed. For example, in 5D EGB theory, causality violation still happens in the presence of the linear scalar field but with an effective mass of the graviton dependence [51-53]. If the effective mass of the graviton is large enough, then there will be no causality violation and hence no constraints for the Gauss-Bonnet coupling. For 4D EGB gravity with a linear axion field, one may expect the same result. We defer these discussions to a future study.

Acknowledgements We would like to thank Chunshan Lin and Qingbing Wang for helpful discussions. This work is partly supported by NSFC (No.11875184). SJS is supported by Mid-career Researcher Program through the National Research Foundation of Korea grant No. NRF-2016R1A2B3007687.

Data Availability Statement This manuscript has no associated data or the data will not be deposited. [Authors' comment: This paper is only about analytical calculation and does not involves numerical computation. So there is no data].

Open Access This article is licensed under a Creative Commons Attribution 4.0 International License, which permits use, sharing, adaptation, distribution and reproduction in any medium or format, as long as you give appropriate credit to the original author(s) and the source, provide a link to the Creative Commons licence, and indicate if changes were made. The images or other third party material in this article are included in the article's Creative Commons licence, unless indicated otherwise in a credit line to the material. If material is not included in the article's Creative Commons licence and your intended use is not permitted by statutory regulation or exceeds the permitted use, you will need to obtain permission directly from the copyright holder. To view a copy of this licence, visit http://creativecomm ons.org/licenses/by/4.0/.

Funded by $\mathrm{SCOAP}^{3}$.

\section{References}

1. J.M. Maldacena, Adv. Theor. Math. Phys. 2, 231 (1998). arXiv:hep-th/9711200

2. S.S. Gubser, I.R. Klebanov, A.M. Polyakov, Phys. Lett. B 428, 105 (1998). arXiv:hep-th/9802109

3. E. Witten, Adv. Theor. Math. Phys. 2, 253 (1998). arXiv:hep-th/9802150

4. Y. Kats, P. Petrov, JHEP 0901, 044 (2009). arXiv:0712.0743 [hepth]

5. M. Brigante, H. Liu, R.C. Myers, S. Shenker, S. Yaida, Phys. Rev. D 77, 126006 (2008). arXiv:0712.0805 [hep-th]

6. M. Brigante, H. Liu, R.C. Myers, S. Shenker, S. Yaida, Phys. Rev. Lett. 100, 191601 (2008). arXiv:0802.3318 [hep-th]

7. X.H. Ge, S.J. Sin, Shear viscosity. JHEP 05, 051 (2009). arXiv:0903.2527

8. R.G. Cai, Y.W. Sun, JHEP 0603, 052 (2008). arXiv:0807.2377 [hep-th]

9. X.H. Ge, Y. Matsuo, F.-W. Shu, S.-J. Sin, T. Tsukioka, JHEP 0810, 009 (2008). arXiv:0808.2354 [hep-th]
10. R.G. Cai, Z.Y. Nie, Y.W. Sun, Phys. Rev. D 78, 126007 (2008). arXiv:0811.1665 [hep-th]

11. R. G. Cai, N. Ohta, Z. Y. Nie, Y. W. Sun, arXiv:0901.1421 [hep-th]

12. A. Buchel, R.C. Myers, JHEP 08, 016 (2009). arXiv:0906.2922

13. D. Glavan, C. Lin, Phys. Rev. Lett. 124(8), 081301 (2020)

14. D. Lovelock, J. Math. Phys. 12, 498 (1971)

15. D. Lovelock, J. Math. Phys. 13, 874 (1972)

16. C. Lanczos, Ann. Math. 39, 842 (1938)

17. S. Sarkar, A.C. Wall, Phys. Rev. D 83, 124048 (2011)

18. R.G. Cai, Phys. Rev. D 65, 084014 (2002). arXiv:hep-th/0109133

19. R.G. Cai, Q. Guo, Phys. Rev. D 69, 104025 (2004). arXiv:hep-th/0311020

20. R.G. Cai, Phys. Lett. B 582, 237 (2004). arXiv:hep-th/0311240

21. H. Lü, Y. Pang, arXiv: 2003.11552 [hep-th]

22. R. A. Konoplya, A. Zhidenko, arXiv:2003.07788 [gr-qc]

23. P.G.S. Fernandes, arXiv:2003.05491 [gr-qc]

24. R. Konoplya, A. Zinhailo, arXiv:2003.01188 [gr-qc]

25. M. Guo, P.C. Li,. arXiv:2003.02523 [gr-qc]

26. S.W. Wei, Y.X. Liu, arXiv:2003.07769 [gr-qc]

27. A. Casalino, A. Colleaux, M. Rinaldi, S. Vicentini,. arXiv:2003.07068 [gr-qc]

28. R. Kumar, S.G. Ghosh,. arXiv:2003.08927 [gr-qc]

29. K. Hegde, A.N. Kumara, C.L.A. Rizwan, A.K.M., M.S. Ali, arXiv:2003.08778 [gr-qc]

30. D.D. Doneva, S.S. Yazadjiev, arXiv:2003.10284 [gr-qc]

31. S.G. Ghosh, S.D. Maharaj, arXiv:2003.09841 [gr-qc]

32. R. Araneda, R. Aros, O. Miskovic, R. Olea, arXiv:1602.07975

33. S. Mahapatra, arXiv: 2004.09214

34. P. G. Fernandes, P. Carrilho, T. Clifton, D. J. Mulryne, arXiv:2004.08362 [gr-qc]

35. D.V. Singh, S. Siwach, arXiv:2003.11754 [gr-qc]

36. D.V. Singh, S.G. Ghosh, S.D. Maharaj, arXiv:2003.14136 [gr-qc]

37. A. Aragón, R. Bécar, P.A. González, Y. Vásquez, arXiv:2004.05632 [gr-qc]

38. D. Samart, P. Channuie, arXiv: 2005.02826 [gr-qc]

39. P. Kovtun, D.T. Son, A.O. Starinets, Phys. Rev. Lett. 94, 111601 (2005). arXiv:hep-th/0405231

40. G. Policastro, D.T. Son, A.O. Starinets, Phys. Rev. Lett. 87, 081601 (2001). arXiv:hep-th/0104066

41. P. Kovtun, D.T. Son, A.O. Starinets, JHEP 0310, 064 (2003). arXiv:hep-th/0309213

42. A. Buchel, J.T. Liu, Phys. Rev. Lett. 93, 090602 (2004). arXiv:hep-th/0311175

43. E. Shuryak, S.-J. Sin, I. Zahed, J. Korean Phys. Soc. 50, 384 (2007). arXiv:hep-th/0511199

44. K.-Y. Kim, S.-J. Sin, I. Zahed, arXiv:hep-th/0608046

45. Rong-Gen Cai, Yan Liu, Ya-Wen Sun, JHEP 1004, 090 (2010)

46. Mohammad Edalati, Juan I. Jottar, Robert G. Leigh, JHEP 1001, $018(2010)$

47. X.H. Ge, S. Sin, S. Wu, G. Yang, Phys. Rev. D 80, 104019 (2009)

48. X.O. Camanho, J.D. Edelstein, J. Maldacena, A. Zhiboedov, JHEP 02, 020 (2016). arXiv: 1407.5597 [hep-th]

49. N. Afkhami-Jeddi, T. Hartman, S. Kundu, A. Tajdini, JHEP 12, 049 (2017)

50. S.D. Chowdhury, A. Gadde, T. Gopalka, I. Halder, L. Janagal, S. Minwalla, JHEP 02, 114 (2020). arXiv:1910.14392 [hep-th]

51. S.A. Hartnoll, D.M. Ramirez, J.E. Santos, J. High Energy Phys. 03, 170 (2016)

52. Y. Wang, X.H. Ge, Phys. Rev. D 94, 066007 (2016)

53. M. Sadeghi, S. Parvizi, Class. Quantum Gravit. 33, 035005 (2016). arXiv:1507.07183 [hep-th] 